\title{
Política andina de planificación y gestión de recursos humanos en salud
}

Andean region policies in planning and management of human resources in health

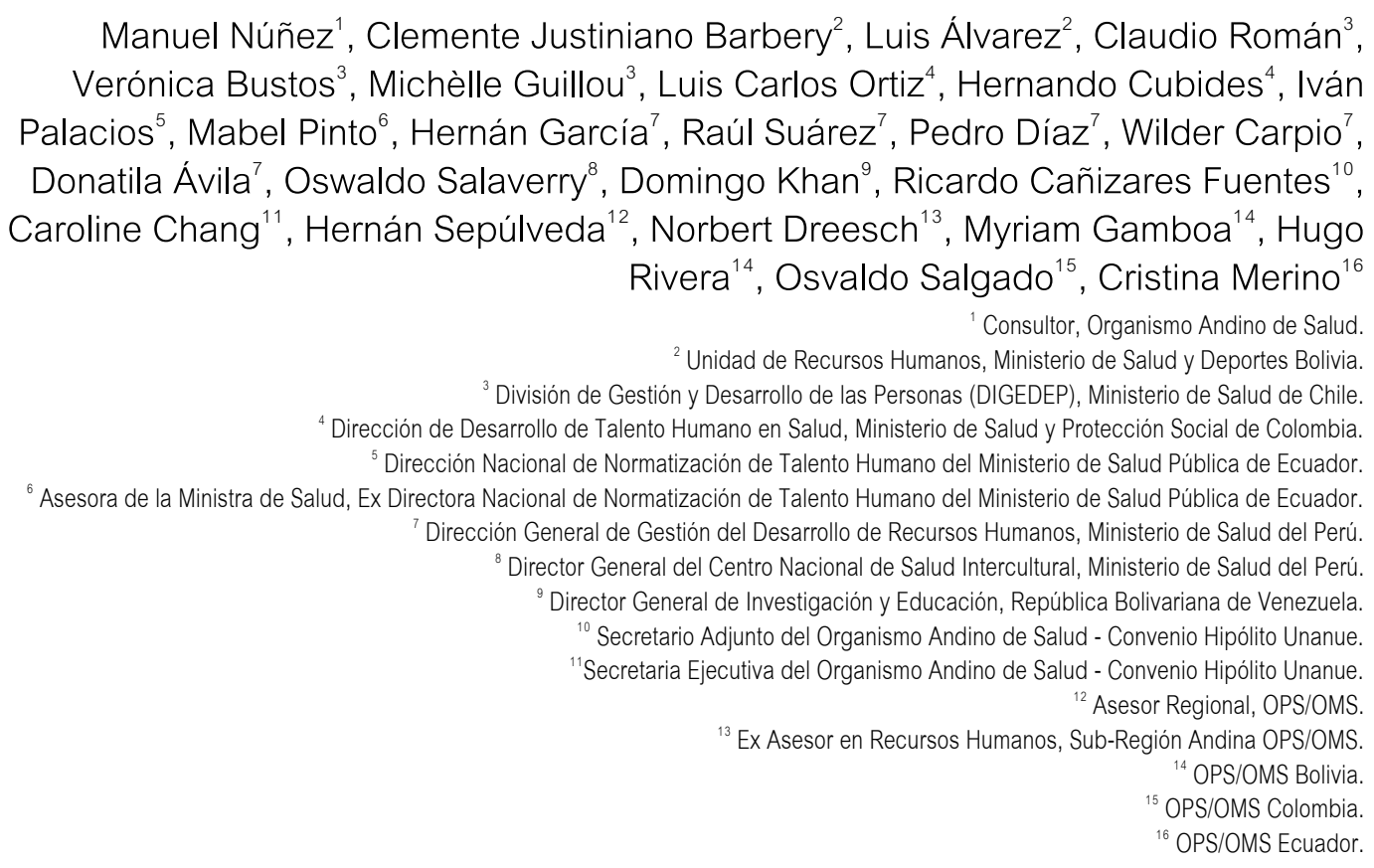

An Fac Med. 2015;76:27-33 / doi:10.15381/anales.v76i1.10967

\section{PRESENTACIÓN}

Los países de la subregión andina: Bolivia, Chile, Colombia, Ecuador, Perú y Venezuela, integrados en el Organismo Andino de Salud - Convenio Hipólito Unanue (ORAS - CONHU) elaboramos y aprobamos mediante Resolución REMSAA XXXIII/474 del 23 de Noviembre de 2012, la Política Andina de Planificación y Gestión de Recursos Humanos en Salud y la implementación de su Plan de Acción 2013 - 2017.

La aprobación de esta política demuestra la visibilidad actual que tiene la problemática de los recursos humanos en salud y el posicionamiento político que está teniendo en la agenda pública nacional y subregional andina y la importancia de ir sumando voluntades políticas multisectoriales para implementar las soluciones más viables.

El nuevo Plan Estratégico del ORAS - CONHU tiene como objetivo trabajar junto a los países andinos para que consoliden sus Sistemas Universales de Salud considerando que es el mejor mecanismo social para garantizar la protección y el goce pleno del derecho a la salud de sus ciudadanos.

Los recursos humanos de salud desempeñan un papel esencial en el funcionamiento adecuado de los sistemas de salud al ser los encargados de proveer la atención de salud con calidad y de acuerdo a las necesidades de salud de toda la población. En ese sentido la política andina y su plan de acción tiene como objetivo servir de referente para los países que están preparando sus políticas nacionales y está alineada a las ya existentes, de tal forma que ayude a avanzar a cada país pero también ayude al conjunto de países andinos para avanzar juntos.

Si bien en los últimos años los países andinos han realizado importantes avances en la planificación y gestión de los recursos humanos, sin embargo, persisten dificultades relacionadas con la falta de personal de 
salud y una inadecuada distribución, se requiere más especialistas clínicos pero al mismo tiempo no hay suficiente personal trabajando en atención primaria. Se necesita suficiente personal para atender problemas de enfermedades transmisibles, pero también personal para atender los nuevos problemas globales como las enfermedades no transmisibles y los afectados por el cambio climático y los desastres.

Para ayudar a resolver estas dificultades la política contiene los temas que los países acordaron trabajar en conjunto en acciones integradas y de largo plazo concretando normativas y acuerdos para consolidar la integración andina en salud.

La política andina tiene 5 líneas estratégicas que abordan los temas de preocupación común de los países andinos:

- Fortalecimiento del rol rector de la autoridad sanitaria en los países andinos.

- Desarrollo de capacidades para sistemas de salud basados en la atención primaria, atención integral, salud familiar e interculturalidad.
- Fortalecimiento de la capacidad de planificación para disminuir la brecha de recursos humanos, incluyendo la gestión de la migración.

- Desarrollo del trabajo decente y competencias para la gestión del trabajo en salud.

- Desarrollo de la inteligencia colectiva en recursos humanos.
Participaron en la elaboración de esta política andina y su plan de acción, el Grupo Técnico Andino de Recursos Humanos en Salud integrado por los delegados de las direcciones nacionales de recursos humanos así como por los técnicos del ORAS - CONHU y OPS/ OMS.

La política Andina de Salud, fue aprobada a través de la Resolución REMSAA NoXXXIII/474 de fecha 23 de Noviembre de 2012.

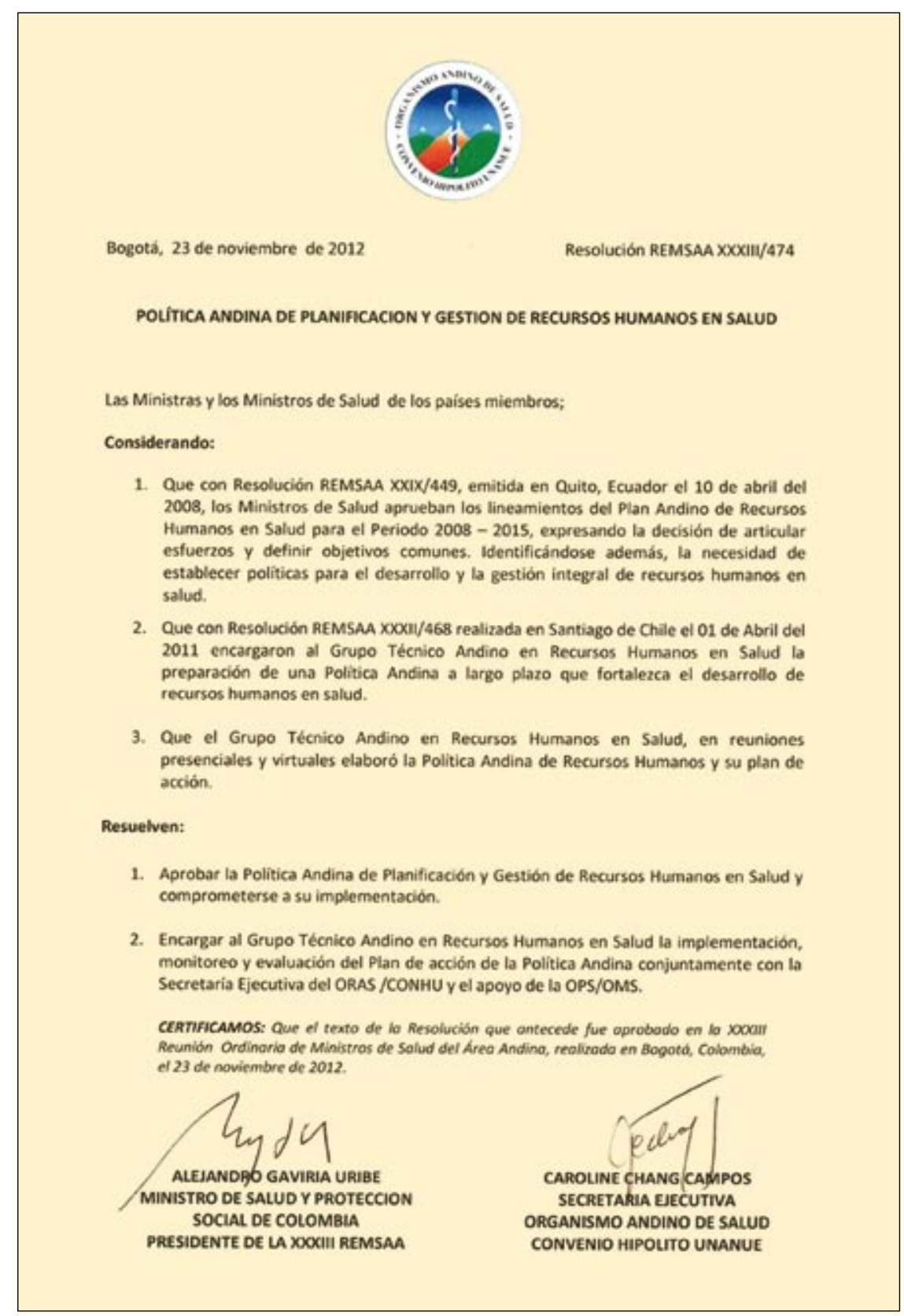




\section{ANTECEDENTES}

Los trabajadores de salud son el pilar fundamental en el funcionamiento de los sistemas sanitarios, los cuales requieren trabajadores competentes y comprometidos, pero a la vez en número suficiente y adecuadamente distribuidos y reconocidos.

Así, el abordaje de los problemas existentes en el campo de los recursos humanos se constituye en condición básica para el desarrollo efectivo de las políticas sanitarias y el resolver estos problemas tema vigente.

Por ello, en los últimos años se ha desarrollado una intensa movilización de alcance nacional e internacional en la región de las Américas, y en particular en los países de la Subregión Andina, para abordar estos problemas, con el fin de garantizar a la población el acceso a servicios de salud con equidad, calidad, eficiencia y pertinencia.

Estas experiencias, particularmente en el campo de los recursos humanos, requieren ser compartidas, convergiendo esfuerzos e iniciativas, definiendo objetivos comunes y aprovechando al máximo nuestras potencialidades y la voluntad de integración.

Importantes iniciativas se han desarrollado con el propósito de generar un trabajo coordinado regionalmente en los temas de recursos humanos, teniendo como un referente importante en la última década, la Resolución CD45.R9 del Consejo Directivo de la Organización Panamericana de la Salud (2004), en la que se insta a los países miembros a que:

- Aborden los problemas persistentes y emergentes en relación con la disponibilidad, la composición, la distribución y el desempeño de los recursos humanos de salud, que constituyen obstáculos importantes a la meta de acceso universal a servicios de salud de buena calidad y al logro de la equidad en materia de salud;
- Ejerzan el liderazgo eficaz mediante el establecimiento de un programa nacional para el desarrollo de recursos humanos, y que promuevan la participación activa de los interesados directos pertinentes en todas las fases de la formulación de políticas;

- Inviertan en el desarrollo de recursos humanos para apoyar la estrategia de atención primaria de salud y el cumplimiento de las funciones esenciales de salud pública, como aportación decisiva para el cumplimiento de los objetivos de la Declaración del Milenio de las Naciones Unidas;

- Exploren maneras de abordar mejor los aspectos complejos de la contratación activa de profesionales de la salud de los países en desarrollo dentro de un marco de emigración ordenada;

- Intensifiquen su participación en el Observatorio de Recursos Humanos de Salud como una estrategia apropiada para definir las prioridades y formular políticas sostenibles.

En esta línea, la VII Reunión Regional de los Observatorios de Recursos Humanos en Salud, efectuada en Toronto - Canadá en Octubre del 2005, aprueba la iniciativa "Llamada a la Acción de Toronto: Hacia una Década de Recursos Humanos en salud para las Américas", que se expresa en los 5 Desafíos de Toronto:

1. Definir políticas y planes de largo plazo para la adecuación de la fuerza de trabajo a las necesidades de salud, a los cambios previstos en los sistemas de salud y desarrollar la capacidad institucional para ponerlas en práctica y revisarlas periódicamente.

2. Colocar las personas adecuadas en los lugares adecuados, consiguiendo una distribución equitativa de los profesionales de salud en las diferentes regiones y de acuerdo con diferentes necesidades de salud de la población.
3. Regular los desplazamientos y migraciones de los trabajadores de salud, de manera que permitan garantizar atención a la salud para toda la población.

4. Generar relaciones laborales entre los trabajadores y las organizaciones de salud que promuevan ambientes de trabajo saludables y permitan el compromiso con la misión institucional de garantizar buenos servicios de salud para toda la población.

5. Desarrollar mecanismos de interacción entre las instituciones de formación (universidades, escuelas) y los servicios de salud que permitan adecuar la formación de los trabajadores de la salud para un modelo de atención universal, equitativo y de calidad, que sirva a las necesidades de salud de la población.

Estos Desafíos y las Metas Regionales aprobadas en Octubre del 2007 por la 27ㄹ Conferencia Sanitaria Panamericana, tienen la virtud de direccionar los cambios a desarrollar en el campo de los recursos humanos, necesarios para que estos tengan un impacto sustancial en el desempeño general de los sistemas de salud, brindando una atención con calidad y equidad.

Paralelamente, la mayoría de los países de la región implementan los Observatorios Nacionales de Recursos Humanos, los cuales se muestran como una estrategia importante para generar información y evidencia que soporte la decisión política, y permita el monitoreo y evaluación participativa de los avances y dificultades encontrados.

Así, los Desafíos de Toronto, las Metas Regionales y el Observatorio de Recursos Humanos, se constituyen en importantes referentes y catalizadores de la construcción de las políticas y estrategias de recursos humanos en los países de la región.

Paralelamente, un esfuerzo convergente de gran importancia es el desarrollado en UNASUR, que en el marco de la integración sudamericana, viene 
implementando importantes iniciativas, como el Instituto Sudamericano de Gobierno en Salud - ISAGS, el Programa de Becas de UNASUR-Salud y la conformación de Redes de Intercambio interinstitucional como estrategia estructurante para el fortalecimiento de los sistemas de salud.

En el ámbito andino, las Ministras y Ministros de Salud de los países miembros del Organismo Andino de Salud, aprueban los lineamientos del Plan Andino de Recursos Humanos en Salud para el Periodo 2008 - 2015 (Resolución REMSAA XXIX/449), expresando la decisión de articular esfuerzos y definir objetivos comunes; decisión que se ratifica en el Plan Estratégico 2009 . 2012, del Organismo Andino de Salud - Convenio Hipólito Unanue (2008), que aprueba el establecer políticas para el desarrollo y la gestión integral de recursos humanos en salud (Objetivo Estratégico 4).

Paralelamente y catalizados por estos acuerdos, los países miembros del Organismo Andino de Salud - Convenio Hipólito Unanue han venido desarrollando importantes iniciativas, con el apoyo de la Organización Panamericana de la Salud, incluyendo los Cursos Internacionales de Gestión de Políticas de Recursos Humanos (CIRHUS), el Observatorio Andino de Recursos Humanos en Salud, investigaciones nacionales y multicéntricas que estudian la migración de profesionales de la salud, y el desarrollo de foros y reuniones que abordan la carrera sanitaria, la gestión de la información y el conocimiento en recursos humanos, así como la convergencia de las agendas regional, subregionales y nacionales, entre otras.

Un momento de confluencia de los esfuerzos desarrollados a nivel nacional, subregional y regional se da entre el 16 y el 19 de noviembre del 2010, con la realización de 3 encuentros coordinados y sucesivos en la ciudad de Lima: a) los puntos focales de la OPS/ OMS de los países de la Sub Región Andina, b) los Directores Nacionales de los países de la Sub Región Andina y, c) los Directores de Recursos Humanos de los Países del ámbito de UNASUR; llegando a concordar las agendas nacionales y sub-regional en la perspectiva del fortalecimiento de la integración ORAS - CONHU/UNASUR/OPS OMS para el desarrollo de los recursos humanos en salud, estructurándose de esta manera una agenda convergente (Organización Panamericana de la Salud 2010).

En abril del 2011, las Ministras y Ministros de Salud de los países andinos, deciden avanzar en la aprobación de la Política Andina de Recursos Humanos, ratificando de esta manera la vigencia plena de este tema en las agendas de los ministerios de salud de la subregión y encargando su preparación al Grupo Técnico de Recursos Humanos.

Para avanzar en este encargo, bajo la conducción del Organismo Andino de Salud y la cooperación de la Organización Panamericana de la Salud, se establece un proceso de recopilación y análisis de experiencias y avances en los países, se implementa un proceso participativo de consultas entre las $\mathrm{Di}$ recciones Nacionales de Recursos $\mathrm{Hu}$ manos sobre los documentos de trabajo, y se genera una propuesta de Política que expresa el consenso alcanzado y que será elevada a la próxima Reunión de Ministras y Ministros de Salud del Área Andina (REMSAA) para su aprobación.

\section{JUSTIFICACIÓN}

Los problemas existentes en el campo de los recursos humanos, expresados en insuficiencia y distribución inequitativa del personal de salud, ausencia o débil presencia de un marco normativo laboral, presencia de importantes grupos de trabajadores en condiciones de empleo precario, desarticulación de instituciones prestadoras y formadoras, pérdida de personal calificado por la migración desregulada, y niveles importantes de conflictividad laboral, entre otros, están presentes en mayor o menor medida en nuestros países andinos, y se constituyen en retos que requieren ser enfrentados, si queremos avanzar en los objetivos sanitarios establecidos.

La necesidad de potenciar la salud y la vida, enfrentar al mismo tiempo una mejoría de resultados sanitarios en las enfermedades transmisibles y la carga creciente de las enfermedades no transmisibles, crónicas y pluripatologías, así como su promoción y prevención, que experimentan en menor o mayor medida los países de la zona andina, constituye un desafío adicional crítico para la Política Andina de Recursos Humanos en Salud.

Así, estos desafíos y el rol crucial que los recursos humanos desempeñan en los sistemas de salud y en los resultados sanitarios, requieren de políticas y estrategias que conjuguen una mirada compartida ante los problemas existentes, nos permitan aprender de las importantes experiencias desarrolladas, articulando esfuerzos en el trabajo conjunto que requerimos fortalecer, permitiéndonos alcanzar los objetivos sanitarios planteados.

Así, la Política Andina de Recursos Humanos expresa, por un lado, la voluntad de los países miembros del Organismo Andino de Salud - Convenio Hipólito Unanue (ORAS-CONHU) de enfrentar juntos los problemas existentes, pero a la vez, representa un momento de acumulación política y técnica de alcance nacional y subregional, que ha de fortalecer y catalizar el desarrollo de las políticas, planes y programas nacionales actualmente en curso, reconociendo las especificidades, pero a la vez construyéndose con base en los principios y valores de equidad, universalidad, participación social, integralidad en el cuidado de la salud y los enfoques familiar, comunitario, de derecho e interculturalidad, que todos compartimos.

\section{PROPÓSITO}

La Política Andina de Recursos Humanos tiene como propósito orientar e impulsar el desarrollo y fortalecimiento de la gestión de los recursos humanos en la 
Subregión Andina, como parte esencial a los esfuerzos nacionales por garantizar el acceso universal a una atención integral, y oportuna de la población de los países miembros, haciendo plenamente vigente el derecho a la salud.

\section{ALCANCE}

Las líneas estratégicas que constituyen la Política Andina de Recursos Humanos son de aplicación en el ámbito de la Subregión Andina y en sus países conformantes y de aplicación para el periodo 2013 - 2017.

\section{OBJETIVO}

Contribuir a que la población de la Subregión Andina disponga de personal sanitario suficiente, adecuadamente distribuido, idóneo, competente, motivado y comprometido con la salud de la población y que reconozca y respete la diversidad cultural existente y las distintas formas de concebir la salud.

\section{LÍNEAS ESTRATÉGICAS DE LA POLÍTICA ANDINA DE RECURSOS HUMANOS.}

Los Ministros y Ministras de salud de la Subregión Andina, considerando la propuesta del Grupo Técnico de Recursos Humanos, definieron 5 líneas estratégicas (Resolución REMSAA XXXII/ 468), que se desarrollan a continuación:

\section{Fortalecimiento del rol rector de la autoridad sanitaria en los países andinos}

- Fortalecer las capacidades de gobierno y gestión de la autoridad sanitaria nacional y subnacional para el desarrollo de políticas públicas de recursos humanos en salud.

- Fortalecer en los Ministerios de Salud de la Subregión Andina, de estructuras organizativas encargadas de la dirección estratégica de la gestión del trabajo y el desarrollo de ca- pacidades de los recursos humanos, intervención en el diseño de las políticas, planes y programas de salud nacionales; así como, en la coordinación intra e intersectorial (sectores de educación, finanzas y trabajo, entre otros).

- Fortalecer las capacidades y calificación de los gestores de políticas de recursos humanos en los niveles nacional y subnacionales.

\section{Desarrollo de capacidades para} sistemas de salud basados en la atención primaria de salud, atención integral, salud familiar e interculturalidad.

- Difundir e intercambiar conocimientos, capacidades y experiencias existentes en la Subregión Andina en la gestión y el desarrollo de los recursos humanos, para la atención primaria, atención integral, salud familiar e interculturalidad.

- Generar, en conjunto con el sector educativo, mecanismos que permitan desarrollar, sistematizar y difundir las mejores experiencias en el desarrollo de los recursos humanos, incluyendo la formación del personal de salud con énfasis en el primer nivel de atención.

- Definir los perfiles y funciones del personal de salud, promoviendo su uso como referente para el diseño de los currículos de los programas de formación y la gestión del personal que labora en el sector salud.

- Desarrollar espacios por parte de la Autoridad Sanitaria para armonizar la formación de recursos humanos con las necesidades y prioridades del sector, generando acuerdos entre los Ministerios de Salud, y las Instituciones formadoras y otros organismos e instituciones pertinentes. Así como generar estrategias para incentivar la inclusión, en los currículos de las facultades de ciencias de la salud, contenidos relacionados con la salud intercultural y el reconocimiento y práctica de la medicina ancestral.
Fortalecimiento de la capacidad de planificación para disminuir la brecha de recursos humanos, incluyendo la gestión de la migración

- Promover en los países andinos la implementación de políticas y estrategias para afrontar las brechas existentes en el personal de salud, incluyendo a los profesionales especialistas, generando esquemas colaborativos e intercambio de experiencias en el abordaje de este desafío.

- Fortalecer en los países la aplicación de metodologías para la estimación de brechas de personal de salud.

- Promover el intercambio de experiencias en la planificación de los recursos humanos en los tres niveles de atención de salud, con prioridad en la atención a poblaciones subatendidas, rurales, indígenas, afrodescendientes y otros grupos étnicos, personas con discapacidad, víctimas de la violencia y de difícil acceso.

- Desarrollar, colaborativamente entre los países de la Subregión Andina, sistemas de monitoreo, reclutamiento y retención para afrontar la migración del personal de salud, conformes con los principios del Código Ético Internacional de Contrataciones de Personal de Salud.

Desarrollo del trabajo decente y competencias para la gestión del trabajo en salud

- Promover y fortalecer normas y mecanismos de vinculación laboral del personal del sector que mejoren progresivamente las condiciones para su desarrollo personal y profesional, incentiven la calidad y continuidad de los servicios de salud, la implementación o fortalecimiento de los programas de salud y seguridad del personal, teniendo como punto de partida el concepto de trabajo decente y como una de las opciones la carrera sanitaria.

- Fortalecer, monitorear y evaluar las estrategias de formación, perfeccionamiento y capacitación continua 
del personal de Salud en un modelo de gestión por competencias, con el objeto de aportar al desarrollo institucional y personal, contribuyendo así al logro de los estándares de calidad del servicio y satisfacción del usuario, la resolución de problemas y el desarrollo de áreas críticas de la organización de salud.

- Intensificar la cooperación técnica entre los países de la sub región respecto del desarrollo del trabajo decente en salud y la carrera sanitaria, generando a la vez experiencias colaborativas en el desarrollo de competencias para la gestión del trabajo del personal de salud.

\section{Desarrollo de una inteligencia colectiva en recursos humanos.}

- Intensificar el desarrollo de un conjunto de datos básicos, comparables y homogéneos sobre la situación de los recursos humanos en salud, con alcance subregional, nacional, subnacional, sectorial e intersectorial, en coordinación con las iniciativas desarrolladas por la Organización Panamericana de la salud, UNASUR y el Instituto Suramericano de Gobierno en Salud (ISAGS).

- Fortalecer la Red Andina de Observatorios de Recursos Humanos en Salud, como instrumento de intercambio de información estratégica.

- Diseminar y promover el desarrollo de investigaciones sobre temas críticos en Recursos Humanos para la subregión.

\section{IMPLEMENTACIÓN Y FINANCIAMIENTO}

Los países de la Subregión Andina se comprometen a la ejecución de esta política, adecuándola en el marco de sus legislaciones nacionales y destinando o consiguiendo el financiamiento necesario, oportuno y sostenido.

Para la implementación de las estrategias señaladas y el desarrollo de los planes operativos, se considerarán ade- más las resoluciones aprobadas por los cuerpo directivos de la OMS, la OPS, el Organismo Andino de Salud/Convenio Hipólito Unanue, y los acuerdos suscritos en el marco de UNASUR y otros espacios de integración.

\section{MONITOREO Y EVALUACIÓN}

El Organismo Andino de Salud - Convenio Hipólito Unanue, a través de su Grupo Técnico de Recursos Humanos en Salud, revisará y reformulará el Plan Andino de Recursos Humanos 2008. 2015, adecuándolo a la Política aprobada, de tal forma que se convierta en su Plan de Acción, el que será puesto a consideración para aprobación de los Ministros y Ministras de Salud del Área Andina, contando para ello con el apoyo técnico de la Organización Panamericana de la Salud y otras entidades de cooperación técnica.

Asimismo, el Organismo Andino de Salud - Convenio Hipólito Unanue deberá coordinar las acciones necesarias que permitan la ejecución del plan de acción de la política por parte de los países, estableciendo un sistema de monitoreo y evaluación, que permita el seguimiento de los acuerdos y decisiones, $\mathrm{y}$ la adopción de correctivos necesarios en función del propósito y objetivo trazado.

Para el diseño y desarrollo de los indicadores de gestión y resultados, se considerarán los lineamientos de OPS/OMS, incluyendo las Metas Regionales para el Desarrollo de los Recursos Humanos 2007 - 2015, aprobadas por la 27 ${ }^{\mathrm{a}}$ Conferencia Sanitaria Panamericana.

\section{PARTICIPARON EN LA CONSTRUC- CION DE ESTE DOCUMENTO}

\section{Bolivia}

- Clemente Justiniano Barbery, Jefe Nacional de la Unidad de Recursos Humanos, Ministerio de Salud y Deportes.

- Luis Álvarez, ex Jefe Nacional de la Unidad de Recursos Humanos, Ministerio de Salud y Deportes.
- Miryam Gamboa, Organización Panamericana de la Salud - Bolivia.

- Hugo de Rivera, Organización Panamericana de la Salud - Bolivia.

Chile

- Claudio Román, Jefe de la División de Gestión y Desarrollo de las Personas (DIGEDEP). Subsecretaria de Redes Asistenciales, Ministerio de Salud.

- Verónica Bustos, Profesional del Departamento de Planificación y Control. DIGEDEP - Subsecretaría de Redes Asistenciales.

- Michèlle Guillou, Profesional del Departamento de Planificación y Control. DIGEDEP - Subsecretaría de Redes Asistenciales.

\section{Colombia}

- Luis Carlos Ortiz, Director de Desarrollo de Talento Humano en Salud, Ministerio de Salud y Protección Social.

- Hernando Cubides, Dirección de Desarrollo de Talento Humano en Salud, Ministerio de Salud y Protección Social.

- Osvaldo Salgado, Organización Panamericana de la Salud - Colombia.

\section{Ecuador}

- Dr. Iván Palacios, Director Nacional de Normatización de Talento Humano del Ministerio de Salud Pública

- Dra. Mabel Pinto, Asesora de la Ministra de Salud, ex Directora Nacional de Normatización de Talento Humano del Ministerio de Salud Pública.

- Dra. Cristina Merino, Organización Panamericana de la Salud - Ecuador.

\section{Perú}

- Hernán García, Director General de Gestión del Desarrollo de Recursos Humanos, Ministerio de Salud. 
- Raúl Suárez, ex Director General de Gestión del Desarrollo de Recursos Humanos, Ministerio de Salud.

- Pedro Díaz, Director Adjunto de la de la Dirección General de Gestión del Desarrollo de Recursos Humanos, Ministerio de Salud.

- Wilder Carpio, ex Director Adjunto de la Dirección General de Gestión del Desarrollo de Recursos Humanos, Ministerio de Salud.

- Donatila Ávila, Coordinadora Técnica de la Dirección General de Gestión del Desarrollo de Recursos Humanos, Ministerio de Salud.

- Oswaldo Salaverry, Director General del Centro Nacional de Salud Intercultural.

\section{Venezuela}

- Domingo Khan, Director General de Investigación y Educación, República Bolivariana de Venezuela.

\section{Organismo Andino de Salud - Convenio Hipólito Unánue (ORAS - CONHU)}

- Caroline Chang, Secretaria Ejecutiva del Organismo Andino de SaludConvenio Hipólito Unanue.

- Ricardo Cañizares, Secretario Adjunto del Organismo Andino de Salud - Convenio Hipólito Unanue.

- Manuel Núñez, Consultor.

\section{Organización Panamericana de la Salud (OPS)}

- Hernán Sepúlveda, Asesor Regional, OPS/OMS.

- Norbert Dreesch, ex Asesor en Recursos Humanos, Sub-región Andina, OPS/OMS.

- Dra. Myriam Gamboa. OPS/OMSBolivia.

- Dr. Hugo Rivera OPS/OMS-Bolivia.

- Dr. Osvaldo Salgado. OPS/OMS. Colombia

- Dra. Cristina Merino. OPS/OMS. Ecuador.

\section{REFERENCIAS BIBLIOGRAFÍCAS}

1. Arroyo J. Estudio del Perfil de las Unidades de Recursos Humanos de los Ministerios de Salud de América Latina y el Caribe. Organización Panamericana de la Salud - Seminario Internacional de Directores de Recursos Humanos de Salud. Septiembre de 2007.

2. Artaza Barrios O, Sacoto Aizaga F, Yglesias Bedoya A, Yánez Monteverda L, Martínez Martín F, Torres-Goitia Torres J. Reformas y Financiamiento de Sistemas y Servicios de Salud en la Subregión Andina. Organismo Andino en Salud-Convenio Hipólito Unanue. Lima, Septiembre de 2004.

3. Asamblea Nacional Constituyente de Venezuela. Constitución de la República Bolivariana de Venezuela. 24 de Marzo de 2000. Disponible en: http:// www.tsj.gov.ve/legislacion/constitucion1999.htm (último acceso: 11 de Enero de 2012).

4. Brito P. El impacto de las reformas del sector salud sobre los recursos humanos y la gestión laboral. Revista Panamericana de Salud Pública. 2000:8(1-2):43-54.

5. Bustíos Romani C. Crisis de los Sistemas de Salud y de Seguridad Social en el Perú: 1968 - 1990. Lima: Facultad de Medicina UNMSM, 2007.

6. Carrasco Dájer C. Programa de Retorno de Médicos Residentes en Chile -Consultoria para obtener las bases científicas a fin de realizar un programa de retorno de personal de salud residente en Chle. Informe Final, Organización Panamericana de la Salud, Quito, 2010.

7. Castellanos J. Recopilación, análisis y sistematización de normas e instrumentos administrativos, con prioridad en medicina y enfermeria (formación y práctica profesional). Bogotá: Organización Panamericana de la Salud, 2010.

8. Castellanos J, Rosales C, Rozas C. El Proceso de Construcción de las Carreras Sanitarias en la Región de las Américas: Análisis de situación y perspectivas de la cooperación técnica. Washington D.C.: Organización Panamericana de la Salud, 2006.

9. Congreso de Colombia. «Ley 1438 de 2011.» 19 de Enero de 2011.

10. Congreso de la República del Perú. «Ley de Aseguramiento Universal en Salud.» Ley Nº 29344. Lima, 2009.

11. Consejo de Salud Sudamericano. «Primera Reunión del Grupo Técnico de Desarrollo y Gestión de Recursos Humanos en Salud del Consejo Suramericano de Salud de UNASUR.» 24 - 25 de Septiembre de 2009.

12. Encuentro Andino de Directores Nacionales de Recursos Humanos de Salud. "Informe y Relatoria» Organización Panamericana de la Salud. Lima 18- 20 de Octubre de 2011.

13. Estado Plurinacional de Bolivia. «Nueva Constitución Política del Estado» 2009.

14. Estado Plurinancional de Boliva. «Decreto Supremo Nº 29601.» Establece el Modelo de Atención y el Modelo de Gestión en Salud en el marco de la Salud Familiar Comunitaria Intercultural - SAFCI. 11 de Junio de 2008

15. Gamero J. Impacto financiero de la emigración de médicos y enfermeras del Área Andina - Un ejercicio de consolidación de documentos país. Lima: Organización Panamericana de la Salud, 2010.

16. Gobierno de Chile. «Estrategia Nacional de Salud. Para el cumplimiento de los Objetivos Sanitarios de la Década 2011 - 2020» Santiago de Chile, 2011.

17. Guillou M, Carabantes J, Bustos V. Disponibilidad de médicos y especialistas en Chile. Rev Med
Chile. 2011;139:559-70.

18. Ministerio de Salud de Chile - División de Gestión y Desarrollo de las Personas. «Descripción del Sistema de Carrera Sanitaria e Incentivos de Chile.» Informe, Santiago de Chile, 2012.

19. Ministerio de Salud de Perú. «Carrera Sanitaria en el Perú. Fundamentos para su Desarrollo.» Serie Bibliográfica - Recursos Humanos en Salud Nº 13. Lima: Dirección General de Gestión del Desarrollo de Recursos Humanos, 2009.

20. Ministerio de Salud y Deportes de Bolivia, «Política de Desarrollo de Recursos Humanos en Salud.» Resolución Ministerial 1233. La Paz, 8 de Diciembre de 2009.

21. Organismo Andino de Salud - Convenio Hipólito Unanue. «Análisis de Situación de Salud Andino, ASIS Andino, con enfoque intercultural.» 2009.

22. Organización Mundial de la Salud. «Informe Mundial de la Salud.» 2006. Disponible en: http://www. who.int/whr/2006/whr06_es.pdf

23. Organización Panamericana de la Salud / Organismo Andino de Salud - Convenio Hipólito Unanue. «Reunión - Migración Calificada de los Recursos Humanos de la Salud en la Subregión Andina.» La Paz, 22 - 24 de Marzo de 2011.

24. Organización Panamericana de la Salud. « $27^{\text {a }}$ Conferencia Panamerica de la Salud - $59^{\text {a }}$ Sesión del Comité Regional.» Metas Regionales en Materia de Recursos Humanos. Washington, D.C., 1 - 5 de Octubre de 2007b.

25. Organización Panamericana de la Salud. «Estudio: Migración de Recursos Humanos en Salud - Subregión Andina.» Lima, 2006.

26. Organización Panamericana de la Salud. «La Agenda Convergente para el Desarrollo de los Recursos Humanos en Salud.» Informe Final: Encuentros de Armonización y Articulación de los Planes de Acción de los Estados Miembros de OPS/OMS, Organismo Andino de Salud - Convenio Hipólito Unanue y la Unión de Naciones Sudamericanas. 16 - 19 de Noviembre de 2010.

27. Organización Panamericana de la Salud. «Metas Regionales en Materia de Recursos Humanos para la Salud 2007 - 2015.» $27^{\text {a }}$ Conferencia Panamericana de la salud. $59^{\text {a }}$ Sesión del Comité Regional. Washington, D.C., EUA, 1-5 de octubre de 2007a.

28. Organización Panamericana de la Salud. Renovación de la atención primaria de salud en las Américas: documento de posición de la Organización Panamericana de la Salud/Organización Mundial de la Salud (OPS/OMS). Washington, D.C.: OPS, 2007.

29. Programa de Apoyo a la Reforma de Salud (PARS) - Ministerio de la Protección Social de Colombia. «Los recursos humanos de la salud en Colombia. Balance, competencias y prospectiva» Proyecto Plan de largo plazo para el desarrollo y fortalecimiento de los recursos. Bogotá, 2007.

30. REMSAA Extraordinaria XXI/1. «Plan Estratégico 2009 - 2015» Videoconferencia, 16 de Diciembre de 2008.

31. República del Ecuador. «Constitución del EcuadorAsamblea Constituyente.» 2008.

32. Sacoto F, Ordoñez G. Estudio de Migración de Médicos Ecuatorianos a Chile. Organización Panamericana de la Salud. Quito, 2007.

33. Salaverry O. Interculturalidad en Salud. Rev Peru Med Exp Salud Publica. 2010:27(1):80-93.

34. Velazco M. Migraciones de Profesionales: Oportunidades para el Desarrollo Compartido. Quito: Organización Panamericana de la Salud, 2010. 